\title{
Three-phase power factor correction technology in electromagnetic transmitter
}

\author{
Qihui* Zhen, Hangbei Liu, Qingyun Di \\ Key Laboratory of Engineering Geomechanics \\ Institute of Geology and Geophysics, CAS \\ Beijing , China
}

\begin{abstract}
Electromagnetic transmitter is an important part of controlled source electromagnetic sounding system. To ensure that the receiver obtains a signal with high SNR (signal to noise ratio) at sufficiently large depth with high accuracy, a large power transmitter is required. In China and abroad, the high-power transmitters are generally bulky, heavy, and has low power transmission. However, the use of three-phase power factor correction technology can solve the above problems. A simple and practical power control strategy was presented in this study. This can achieve the power factor of one.
\end{abstract}

Keywords - Electromagnetic transmitter, three-phase power factor, correction technology

\section{INTRODUCTION}

Currently, there are three main Electromagnetic sounding transmitter systems, namely; V8 electromagnetic transmitter TXU-30 (Canadian Phoenix), GDP-32 multifunctional transmitter GGT-30 (US ZONGE) and TXM-22 transmitter (German Metronix). In TXU-30 system, uncontrolled rectifier bridge is used to rectify, while PWM (pulse width modulation) DC/DC convertor is used as voltage regulator; GGT-30 transmitter on the other hand achieves its output voltage by selecting transformer gear, while the phase-controlled rectifier bridge is used as voltage regulator. Also, the TXM-22 transmitter uses an uncontrolled rectifier. Power transformers are used for both TXU-30 and GGT-30 systems. This makes the instrument bulky, thus, weighting between 50-100 kilograms. The lighter TXM-22 on the other end, has no adjustable output volt. Their power can only reach $20-30 \mathrm{~kW}$.

The proposed three-phase power factor correction technology uses three boost inductors instead of a transformer, and the voltage is adjustable. This reduces the size of the transmitter, improves its power output, and enhances the utilization rate of electrical energy. This is the ideal solution for electromagnetic transmitter.

\section{Theory and Method}

Fig. 1 shows the circuit topology.

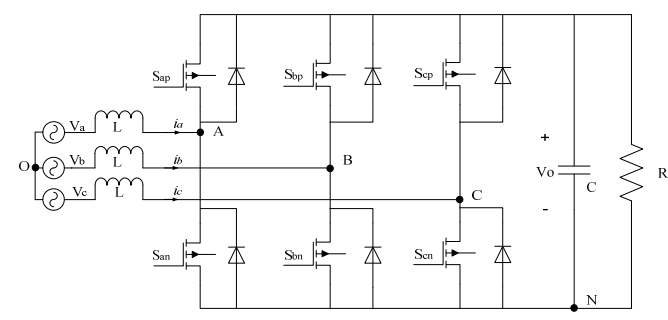

Fig. 1 Circuit topology
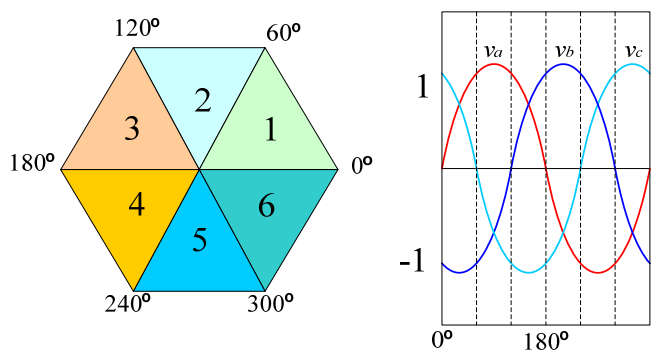

Fig. 2 Voltage partition

The voltage vector was divided into six sectors within one cycle of input volt, as shown in Fig. 2. Three-phase PFC converter can be achieved by controlling the switching state of the two phases within one cycle.

Fig. 2 shows that the maximum phase voltage always have an opposite polarity with respect to the other two phases. Hence, the current of the two phases with the same polarity are controlled to follow the phase voltage, while the third phase switches are kept in an on or off state within each section, such that, the sum of the three-phase current will equal zero.

In order to facilitate the research, we need the following assumptions:

- $\quad$ The generator outputs three-phase voltage, which are symmetrical to each other. The phases are at $120^{\circ}$ to each other, and has no internal resistance ;

- $\quad$ Each phase has equal inductance;

- $\quad$ Switching frequency is much greater than the power frequency; 
- $\quad$ The capacitor is large enough to ensure that the output voltage is constant;

- $\quad$ The dropout voltage and the losses of the switching devices are igfpred $=d V$

Then, the volts of node $\mathrm{A}, \mathrm{B}$ and ${ }^{\circ} \mathrm{C}$ relative to the node $\mathrm{N}$ are:

$$
\left\{v_{B N}=d_{b p} V_{o}\right.
$$

Where $d_{\mathrm{ap}}, d_{\mathrm{bp}}$, and $d_{\mathrm{cp}}$ are the duty cycle of Switches $\mathrm{S}_{\mathrm{ap}}, \mathrm{S}_{\mathrm{bp}}$, and $\mathrm{S}_{\mathrm{cp}}$, respective $Y_{y N a} \overline{\bar{n}}{ }_{d} d_{\phi_{0}} V_{\text {ois }}$ the output voltage. The equivalent average circuit model is shown in Fig. 3.

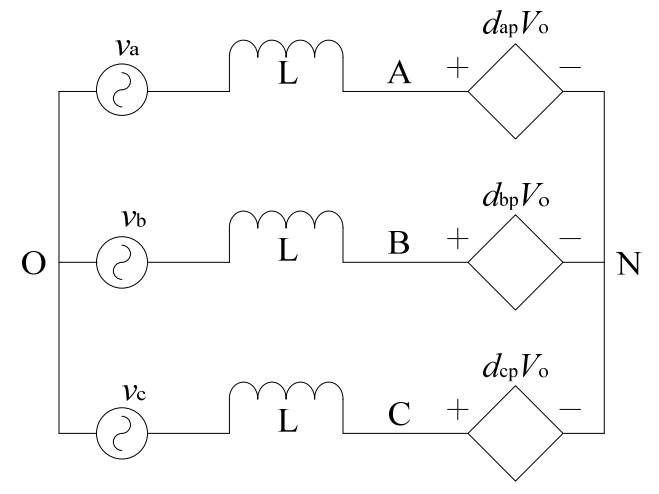

Fig. 3 Equivalent average circuit model

Hence, the volts of node $\mathrm{A}, \mathrm{B}$ and $\mathrm{C}$ relative to the node $\mathrm{O}$ are:

$$
\left\{\begin{array}{l}
v_{A O}=v_{a}-j \omega L i_{a} \\
v_{B O}=v_{a}-j \omega L i_{b} \\
v_{C O}=v_{a}-j \omega L i_{c}
\end{array}\right.
$$

If the three phases are without neutral system, one finds:

$$
\left\{\begin{array}{l}
v_{a}+v_{b}+v_{c}=0 \\
i_{a}+i_{b}+i_{c}=0
\end{array}\right.
$$

Substituting equation (3) into (2) to obtain:

$$
v_{A O}+v_{B O}+v_{C O}=0
$$

The rectifier topology in Fig. 1 shows that:

$$
\left\{\begin{array}{l}
v_{A O}=v_{A N}+v_{N O} \\
v_{B O}=v_{B N}+v_{N O} \\
v_{C O}=v_{C N}+v_{N O}
\end{array}\right.
$$

By combining formula (4) and (5), we obtained:

$$
v_{N O}=-\frac{1}{3}\left(v_{A N}+v_{B N}+v_{C N}\right)
$$

$$
\left\{\begin{array}{l}
v_{A O}=\frac{1}{3}\left(2 v_{A N}-v_{B N}-v_{C N}\right) \\
v_{B O}=\frac{1}{3}\left(2 v_{B N}-v_{A N}-v_{C N}\right) \\
v_{C O}=\frac{1}{3}\left(2 v_{C N}-v_{B N}-v_{A N}\right)
\end{array}\right.
$$

Substituting equation (1) into (7) to obtain:

$$
\left[\begin{array}{ccc}
\frac{2}{3} & -\frac{1}{3} & -\frac{1}{3} \\
-\frac{1}{3} & \frac{2}{3} & -\frac{1}{3} \\
-\frac{1}{3} & -\frac{1}{3} & \frac{2}{3}
\end{array}\right] \cdot\left[\begin{array}{l}
d_{a p} \\
d_{b p} \\
d_{c p}
\end{array}\right]=\frac{1}{V_{o}}\left[\begin{array}{l}
v_{A O} \\
v_{B O} \\
v_{C O}
\end{array}\right]
$$

In section $\left[0^{\circ}, 60^{\circ}\right]$ and from Fig. 3, switch San and Scn are controlled while other switches are kept off, in which case we can get the Equivalent duty cycle:

$$
\left\{\begin{array}{l}
d_{a p}=1-d_{a n} \\
d_{b p}=1 \\
d_{c p}=1-d_{c n}
\end{array}\right.
$$

The goal of power factor correction is to control the three-phase rectifier with inductor current to follow its phase voltage, in which case we can write:

$$
\left\{\begin{array}{l}
v_{a}=R_{e} \cdot i_{a} \\
v_{b}=R_{e} \cdot i_{b} \\
v_{c}=R_{e} \cdot i_{c}
\end{array}\right.
$$

Where $\mathrm{Re}$ is the equivalent resistance of the voltage detection. Since the switching frequency is much greater than the supply voltage frequency, the inductance value is usually relatively small, so the voltage across the inductor is negligible, then, we simply have:

$$
\left\{\begin{array}{l}
v_{A O} \approx v_{a} \\
v_{B O} \approx v_{b} \\
v_{C O} \approx v_{c}
\end{array}\right.
$$

Substituting equation (9), (10), and (11) into (8) to obtain:

Then 


$$
\left[\begin{array}{cc}
\frac{2}{3} & -\frac{1}{3} \\
-\frac{1}{3} & \frac{2}{3}
\end{array}\right] \cdot\left[\begin{array}{l}
1-d_{a n} \\
1-d_{c n}
\end{array}\right]=\frac{R_{e}}{V_{o}}\left[\begin{array}{l}
i_{a} \\
i_{c}
\end{array}\right]
$$

The solution to equation (12) above is given as:

$$
\begin{aligned}
{\left[\begin{array}{l}
1-d_{a n} \\
1-d_{c n}
\end{array}\right] } & =\frac{R_{e}}{V_{o}}\left[\begin{array}{ll}
2 & 1 \\
1 & 2
\end{array}\right] \cdot\left[\begin{array}{l}
i_{a} \\
i_{c}
\end{array}\right] \\
& =\frac{R_{e}}{V_{o} \cdot R_{s}}\left[\begin{array}{ll}
2 & 1 \\
1 & 2
\end{array}\right]\left[\begin{array}{l}
R_{s} \cdot i_{a} \\
R_{s} \cdot i_{c}
\end{array}\right]
\end{aligned}
$$

Where Rs is the equivalent resistance of the current detection. If we consider the definition;

$$
V_{m}=\frac{V_{o} \cdot R_{s}}{R_{e}}
$$

Then,

$$
V_{m}\left[\begin{array}{l}
1-d_{a n} \\
1-d_{c n}
\end{array}\right]=R_{s}\left[\begin{array}{ll}
2 & 1 \\
1 & 2
\end{array}\right] \cdot\left[\begin{array}{l}
i_{a} \\
i_{c}
\end{array}\right]
$$

Similarly, you can control the relation in other sections like in formula (15). The control algorithm obtained (formula (15)) can be used to control two of the six switches and can achieve power factor correction boost function.

\section{SIMULATION EXPERIMENT}

According to equation (15), the block diagram shown in Fig. 4 represents the controller for three-phase power factor correction rectifier.

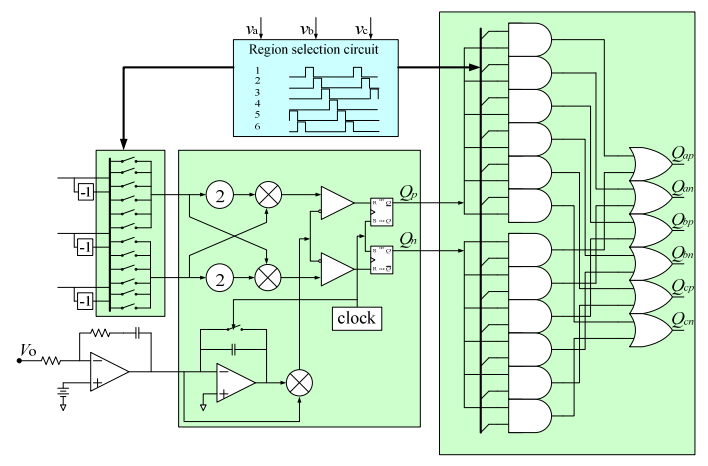

Fig. 4 PFC controller for three-phase boost rectifier

The saber software was used to simulate the PFC system with $220 / 50 \mathrm{~Hz}$ input voltage, $1000 \mathrm{~V}$ output DC voltage and $50 \mathrm{~kW}$ power output. Considering an inductance of $1 \mathrm{mH}$ and clock's frequency of $10 \mathrm{KHz}$, the results obtained are shown in Fig. 5.

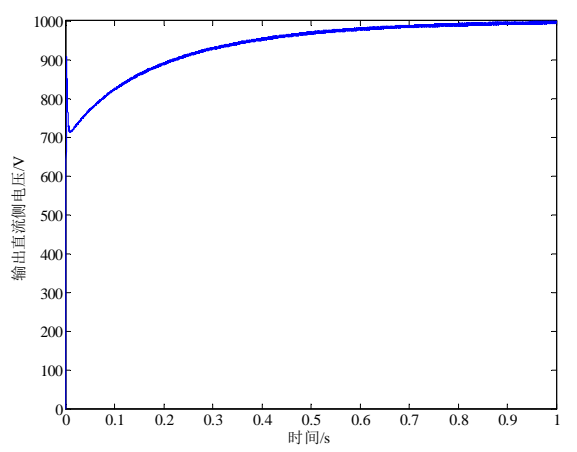

(a)

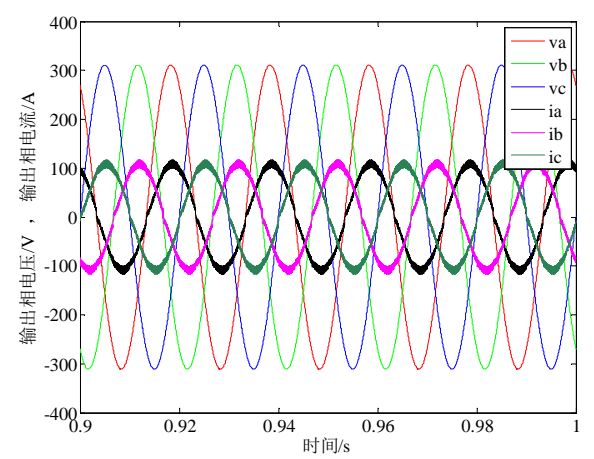

(b)

Fig. 5 Simulated waveforms(a) Output DC volt(b) input three-phase current and volt waveforms

As shown in Fig. 5, Va, Vb, and Vc are the three-phase input voltage waveforms, while ia, ib and ic are the three-phase input current waveforms.

The power factor correction and boost rectifier were achieved by the controller shown in Fig. 4 .

\section{CONCLUSION}

The three-phase PFC technology, which greatly reduces the size and weight of the transmitter, and increases the power output, is the ideal solution for high-power electromagnetic transmitter.

\section{REFERENCES}

[1] Chongming Qiao, Smedley, K.M. 2002. A general three-phase PFC controller for rectifiers with a parallel-connected dual boost topology. Power Electronics, IEEE Transactions on. NO.6, 925-934.

[2] Chongming Qiao, Smedley, K.M. 2002. A general three-phase PFC controller for rectifiers with a series-connected dual boost topology. Power Electronics, IEEE Transactions on Industry Applications. NO.1, 137-14.

[3] Lai Z, Smedley K M. 1996. A new extension of one-cycle control and its application to switching power amplifiers [J]. Power Electronics, IEEE Transactions on. 11(1):99 - 105.

[4] Smith Jr K M, Smedley K M. 2001. Engineering design of lossless 
passive soft switching methods for PWM converters. I. With minimum voltage stress circuit cells [J]. Power Electronics, IEEE Transactions on. 16(3): 336-344.

[5] Smith Jr K M, Smedley K M. 2002. Engineering design of lossless passive soft switching methods for PWM converters. II. With nonminimum voltage stress circuit cells [J]. Power Electronics, IEEE Transactions on. 17(6): 864-873. 